Honam Mathematical J. 35 (2013), No. 1, pp. 073-082

http://dx.doi.org/10.5831/HMJ.2013.35.1.73

\title{
CERTAIN IDENTITIES OF ZETA FUNCTIONS ON A QUATERNION ALGEBRA
}

\author{
INSUK KIM* AND SUNGTAE JUN ${ }^{\dagger}$
}

\begin{abstract}
There are some identities between the representations of Hecke operators defined on a subspace of modular forms associated with orders of a Quaternion algebra. In this paper, we find the identities between the zeta functions attached to these representations.
\end{abstract}

\section{Introduction}

An order $M$ of a quaternion algebra $A$ over a local field $k$ is called primitive if it satisfies one of following conditions. If $A$ is a division algebra, $M$ contains the full ring of integers of a quadratic extension field of $k$. If $A$ is isomorphic to $\operatorname{Mat}_{2 \times 2}(k)$, then $M$ contains a subring which is isomorphic either to $\mathcal{O} \oplus \mathcal{O}$, where $\mathcal{O}$ is the ring of integers in $k$ or to the full ring of integers in a quadratic extension field of $k$. In [3] and [5], a certain subspace of modular forms was studied by using the arithmetic theory of orders in quaternion algebras. Since Brandt matrices $B(n)$ and Hecke Operators $T(n)$ generate semi simple commutative rings, if traces of Brandt matrices and Hecke operators are identical, then Brandt matrices give a representation of the Hecke operators on a space generated by theta series. In [8], Shimizu investigated some arithmetic properties of orders in a quaternion algebra and he found some identities between the zeta functions defined on different orders on quaternion algebras. In order to prove the identities, he investigated the trace formula of Hecke operators which were used to define the zeta functions. Hence, there are some similarities between the work [3] and [8]. In this paper, as an application of [4], we will find some identities between Brandt matrices

Received February 1, 2013. Accepted February 8, 2013.

2010 Mathematics Subject Classification. 11R11.

Key words and phrases. zeta function, order, quaternion algebra, hecke ring.

$\dagger$ This work was supported by Konkuk University in 2010.

* Corresponding author 
and zeta functions defined with these matrices, which are generalizations of $[8]$.

\section{Orders and Brandt matrices}

In this section, we summerize how to construct Brandt matrices with orders. For the computational convenience, we restrict ourselves that $A$ is a rational quaternion algebra ramified precisely at a prime $q$ and $\infty$. That is, $A_{q}=A \otimes \mathbb{Q}_{q}$ and $A_{\infty}=A \otimes \mathbb{R}$ are division algebras where $\mathbb{Q}_{p}$ is a local field. On the other hand, $A_{p}=A \otimes \mathbb{Q}_{p}$ is isomorphic to $M_{2 \times 2}\left(\mathbb{Q}_{p}\right)$ for a finite prime $p \neq q$.

Let $L(p)$ be a quadratic extension field of $\mathbb{Q}_{p}$. It is well known that $\left\{\left(\begin{array}{cc}\alpha & \beta \\ \bar{\beta} & \bar{\alpha}\end{array}\right) \mid \alpha, \beta \in L\right\}$ is a quaternion algebra over $\mathbb{Q}_{p}$ which is isomorphic to $M_{2 \times 2}\left(\mathbb{Q}_{p}\right)$.

Let $A_{p}=\left\{\left(\begin{array}{cc}\alpha & \beta \\ \bar{\beta} & \bar{\alpha}\end{array}\right) \mid \alpha, \beta \in L\right\}=L+\xi L$, where $\xi=\left(\begin{array}{ll}0 & 1 \\ 1 & 0\end{array}\right)$ and $\alpha \in L$ is identical with $\left(\begin{array}{cc}\alpha & 0 \\ 0 & \bar{\alpha}\end{array}\right)$ in $A_{p}$. Hence, we can define the norm of an element in $A$ as its determinant.

Let $P_{L}$ be the prime ideal of $\mathcal{O}_{L}$, the ring of integers in $L=L(p)$. In [5], we have proved that the possibilities of an order, $\Lambda$, of $A_{p}$ containing $\mathcal{O}_{L}$ are as follows:

$$
\Lambda=\left\{\begin{array}{l}
\Lambda_{2 \nu}(L)=\mathcal{O}_{L}+\xi P_{L}^{\nu} \quad \text { if } L \text { is unramified, or } \\
\Lambda_{\nu}(L)=\mathcal{O}_{L}+(1+\xi) P_{L}^{\nu-1} \quad \text { if } L \text { is ramified, or } \\
\Lambda_{0}(L)=\mathcal{O}_{L}+(1-\xi) P_{L}^{-1} \quad \text { if } L \text { is ramified }
\end{array}\right.
$$

for some nonnegative integer $\nu$, where $\xi$ is as above. Hence the level of order $M$ of $A$ can be defined.

Definition 1. Let $A$ be a quaternion algebra ramified precisely at one finite prime $q$ and $\infty$. For finite odd primes, $p_{1}, p_{2}, \cdots p_{d} \neq q$, an order $M$ has level $\left(q ; L\left(p_{1}\right), \nu\left(p_{1}\right) ; \cdots ; L\left(p_{d}\right), \nu\left(p_{d}\right)\right)$ if

(i) $M_{q}$ is the maximal order of $A_{q}$.

(ii) for a prime $p \neq q$, there exists a quadratic extension field $L(p)$ of $Q_{p}$ and nonnegative integer $\nu(p)$ (which is even if $L(p)$ is unramified) such that $M_{p}=R_{\nu(p)}(L(p))$.

(iii) $\nu\left(p_{i}\right)>0$ for $i=1,2 \cdots d$ and $\nu(p)=0$ for $p \neq q, p_{1} \cdots, p_{d}$. (i.e $M_{p}$ is a maximal order of $A_{p}$ if $\left.p \neq p_{1}, p_{2}, \cdots p_{d}\right)$. 
From now on, let $A$ be a quaternion algebra ramified precisely at the odd prime $q$ and $\infty$. Here, we will restrict ourselves with an order $\mathcal{O}$ in a quaternion algebra which has level $N^{\prime}=(q ; L(p), \nu(p))$ with $\nu(p)>1$, where $L(p)$ is a quadratic extension field of $\mathbb{Q}_{p}$.

Let $\mathcal{O}$ be an order of level $N^{\prime}$ in $A$. A left $\mathcal{O}$ ideal $I$ is a lattice on $A$ such that $I_{p}=\mathcal{O}_{p} a_{p}$ (for some $a_{p} \in A_{p}^{\times}$) for all $p<\infty$. Two left $\mathcal{O}$ ideals $I$ and $J$ are said to belong to the same class if $I=J a$ for some $a \in A^{\times}$. Analogously, right $\mathcal{O}$ ideals can be defined. The class number of left ideals for any order $\mathcal{O}$ of level $N^{\prime}$ is the number of distinct classes of such ideals as usual sense.

The norm of an ideal, denoted by $N(I)$, is the positive rational number which generates the fractional ideal of $\mathbb{Q}$ generated by $\{N(a) \mid a \in I\}$. The conjugate of an ideal $I$, denoted by $\bar{I}$, is given by $\bar{I}=\{\bar{a} \mid a \in I\}$ and the inverse of an ideal, denoted by $I^{-1}$, is given by $I^{-1}=\{a \in A \mid I a I \subset$ $I\}$.

Proposition 2.1. Let $\mathcal{O}$ be an order of level $N^{\prime}$ in $A$. Let $I_{1}, I_{2}, I_{3}, \cdots$, $I_{H}$ be the complete set of representatives of all the distinct left $\mathcal{O}$ ideal classes. Let $\mathcal{O}_{j}$ be the right order of $I_{j}, j=1,2, \cdots, H$. Then $I_{j}^{-1} I_{1}, \cdots, I_{j}^{-1} I_{H}$ is a complete set of representatives of all the distinct left $\mathcal{O}$ ideal classes (for $j=1,2, \cdots, H$ ).

Proof. See Proposition 2.13 and 2.15 of [6].

There are several literatures dealt with the definition of Brandt matrices with an order of a quaternion algebra. For a nonnegative integer $n$, we define the generalized Brandt matrices $B(n)=B\left(n ; N^{\prime}\right)$ associated with an order $\mathcal{O}$, in the same manner as Eichler's (See [1], equation 15 and 15a on the page 105). For the details, see [1], [3] or [5].

Let $\mathcal{O}$ be an order of $A$ with level $N^{\prime}$ and let $I_{1}, I_{2}, \cdots, I_{H}$ be a representatives of (left) ideal classes of $\mathcal{O}$ with $H$, the class number. Then let $e_{j}$ be the number of unit elements in $\mathcal{O}_{j}$, the right order of $I_{j}$. The entries of Brandt matrices are defined as $b_{i j}(n)=\frac{1}{e_{j}}$ the number of elements in $I_{j}^{-1} I_{i}$ with norm $n N\left(I_{i}\right) / N\left(I_{j}\right)$ for $n \geq 1$. If $n=0$, $b_{i j}(0)=\frac{1}{e_{j}}$. Then $B\left(n, N^{\prime}\right)=\left(b_{i j}(n)\right)$ is called a Brandt matrix, which is a $H \times H$ matrix.

\section{Hecke operators}

3.1. In the previous section, we defined Brandt matrices. We are now explain how they are related with Hecke operators. Fix an order $\mathcal{O}$ 
in $A$ of level $N^{\prime}=(q ; L(p), \nu(p))$. Let $J_{A}$ denote the idele group of $A$

$$
J_{A}=\left\{\hat{a}=\left(a_{l}\right) \in \prod_{l} A_{l}^{\times} \mid a_{l} \in \mathcal{O}_{l}^{\times} \text {for almost all } l\right\},
$$

where the product is over all $l$ finite and infinite. Via the local global correspondence, $J_{A}$ acts transitively on the left $\mathcal{O}$ ideals.

Let $U=U(\mathcal{O})=\left\{\hat{u}=\left(u_{l}\right) \in J_{A} \mid u_{l} \in \mathcal{O}_{l}^{\times}\right.$for all $\left.l<\infty\right\}$. Since $\hat{a} U \hat{a}^{-1}$ is commensurable with $U$ for all $\hat{a} \in J_{A}$, Hecke ring $R\left(U, J_{A}\right)$ can be defined as the free $\mathbb{Z}$-module generated by all double cosets $U \hat{a} U$, $\hat{a} \in J_{A}$ with multiplication defined as in [9].

Let $J_{\mathbb{Q}}$ denote the idele group of $\mathbb{Q}$ and put $U(\mathbb{Z})=\left\{\hat{u}=\left(u_{l}\right) \in\right.$ $J_{\mathbb{Q}} \mid u_{l} \in \mathbb{Z}_{l}^{\times}$for all $\left.l<\infty\right\}$. The reduced norm $N: A \rightarrow \mathbb{Q}$ induces the reduced norm, $N: J_{A} \rightarrow J_{\mathbb{Q}}$. For a positive integer $n$, we denote by $T(n)$ the element of $R\left(U, J_{A}\right)$ which is the sum of all double cosets $U \hat{a} U$ such that the left ideal $\mathcal{O} \hat{a}$ is integral and of norm $n$. That is, $a_{l} \in \mathcal{O}_{l}$ for all $l<\infty$ and $N(\hat{a}) \in n U(\mathbb{Z})$, where $\hat{a}=\left(a_{l}\right)$.

Let $D_{N}=D_{N}(\mathcal{O})=\left\{\hat{a}=\left(a_{l}\right) \in J_{A}\left|a_{l} \in \mathcal{O}_{l}, \forall l\right| N\right\}$ and let $R\left(U, D_{N}\right)$ denote the subring of $R\left(U, J_{A}\right)$ generated by all $U \hat{a} U$ with $\hat{a} \in D_{N}$. Then $T(n)$ belongs to $R\left(U, D_{N}\right)$ and it is called a Hecke operator.

3.2. We are now in position to explain the space of automorphic functions which Hecke operators act on. For this, we modify the idea in section 5 in [3] to our case. Fix an isomorphism $A_{\infty} \otimes \mathbb{C}=\left(A_{\infty} \otimes_{\mathbb{Q}} \mathbb{R}\right) \otimes$ $\mathbb{C} \simeq \operatorname{Mat}_{2}(\mathbb{C})$ which gives a natural embedding of $A_{\infty}^{\times}$into $G L_{2}(\mathbb{C})$. Let $\psi$ denote the projection $D_{N} \rightarrow A_{\infty}^{\times}$and this embedding $A_{\infty}^{\times} \subset G L_{2}(\mathbb{C})$.

Denote by $\varphi$ the mapping given by

$$
\varphi(\hat{x})=\psi(\hat{x})
$$

for $\hat{x} \in D_{N}$. Note that $\varphi$ is an antihomomorphism from $D_{N}^{\times}$to $G L(\mathbb{C})$.

It is well known that

$$
J_{A}=\cup_{\lambda=1}^{h} U \hat{x_{\lambda}} A^{\times},
$$

where $h$ is the class number of $\mathcal{O}$.

3.3. The double coset representative $\hat{x}_{\lambda}=\left(x_{\lambda l}\right)$ can be chosen so that $x_{\lambda l} \in \mathcal{O}_{l}^{\times}$for all $l \mid N$. For $\lambda=1, \cdots, h$ let $I_{\lambda}=\mathcal{O} \hat{x}_{\lambda}, \mathcal{O}_{\lambda}=\hat{x}_{\lambda}^{-1} \mathcal{O} \hat{x}_{\lambda}$, $U_{\lambda}=\hat{x}_{\lambda}^{-1} U \hat{x}_{\lambda}$ and $U_{\lambda}=U_{\lambda} \cap A^{\times}$. Then all $I_{\lambda}$ are representatives of all the distinct left $\mathcal{O}$-ideal classes, $\mathcal{O}_{\lambda}$ is the right order of $I_{\lambda}$ and $U_{\lambda}=\mathcal{O}_{\lambda}^{\times}$ is the unit group of $\mathcal{O}_{\lambda}$. 
Denote by $M_{2}(\mathcal{O})$ the complex vector space of all continuous functions $f(x)$ on $J_{A}$, taking values in $F$, which satisfy

$$
f(u x \alpha)=\varphi(\alpha)^{-1} f(x)
$$

for all $u \in U, x \in J_{A}$, and $\alpha \in A^{\times}$.

We define a representation of the Hecke ring $R\left(U, D_{N}\right)$ on $M_{2}(\mathcal{O})$ as follows. For a double coset $U y U \in R\left(U, D_{N}\right)$, let $U y U=\cup_{i} U y_{i}$ be its decomposition into disjoint right cosets.

We denote by $\rho(U y U)$ the operator defined by

$$
\rho(U y U) f(x)=g(x), \text { where } g(x)=\sum_{i} \varphi\left(y_{i}\right) f\left(y_{i} x\right) .
$$

It is easy to see that $\rho(U y U)$ is an endomorphism on all of $R\left(U, D_{N}\right)$ by linearity.

Lemma 3.1. $M_{2}(\mathcal{O})$ is an $R\left(U, D_{N}\right)$ module and the structure of $M_{2}(\mathcal{O})$ is independent of the particular choice of $\mathcal{O}$.

Proof. Let $\mathcal{O}^{\prime}$ be another order in $A$ having the same level as $\mathcal{O}$. Since $\mathcal{O}_{l}^{\prime}$ is isomorphic to $\mathcal{O}_{l}$ for all $l<\infty$, let $\mathcal{O}^{\prime}=\hat{\beta} \mathcal{O} \hat{\beta}^{-1}$ where $\hat{\beta}=\left(b_{l}\right) \in J_{A}$ with $b_{\infty}=1$. Then $U\left(\mathcal{O}^{\prime}\right)=\hat{\beta} U(\mathcal{O}) \hat{\beta}^{-1}$ and $D_{N}\left(\mathcal{O}^{\prime}\right)=\hat{\beta} D_{N}(\mathcal{O}) \hat{\beta}^{-1}$. The map $\psi: f(x) \rightarrow f(\hat{\beta} x)$ induces a complex vector space isomorphism of $M_{2}\left(\mathcal{O}^{\prime}\right)$ onto $M_{2}\left(\mathcal{O}^{\prime}\right)$. Then $M_{2}\left(\mathcal{O}^{\prime}\right)$ is isomorphic to $M_{2}(\mathcal{O})$ as Hecke modules.

Clearly the map $U(\mathcal{O}) y U(\mathcal{O}) \rightarrow U\left(\mathcal{O}^{\prime}\right) \hat{\beta} y \hat{\beta}^{-1} U\left(\mathcal{O}^{\prime}\right)$ induces an isomorphism of $R\left(U(\mathcal{O}), D_{N}(\mathcal{O})\right)$ onto $R\left(U\left(\mathcal{O}^{\prime}\right), D_{N}\left(\mathcal{O}^{\prime}\right)\right)$ and view $M_{2}\left(\mathcal{O}^{\prime}\right)$ as an $R\left(U(\mathcal{O}), D_{N}(\mathcal{O})\right)$ module by defining

$$
(U(\mathcal{O}) y U(\mathcal{O})) f(x)=\left(U\left(\mathcal{O}^{\prime}\right) \hat{\beta} y \hat{\beta}^{-1} U\left(\mathcal{O}^{\prime}\right)\right) f(x)
$$

for $f(x) \in M_{2}\left(\mathcal{O}^{\prime}\right)$ and extending by linearity. It follows by an easy calculation that $\psi$ is in fact an $R\left(U(\mathcal{O}), D_{N}(\mathcal{O})\right)$ module isomorphism.

Remark. In [5], we have proved that Brandt matrices generate a set of theta series which are modular forms of weight 2. Also, Brandt matrices are the representation matrices of Hecke operators acting on a subspace of these modular forms.

Next, we like to explain the representative theoretic approach to Brandt matrices.

3.4. Elements of $M_{2}(\mathcal{O})$ are completely determined by their values at $\hat{x}_{\lambda}, \lambda=1, \cdots, h$. In fact for $f \in M_{2}(\mathcal{O})$ put $f_{\lambda}=\varphi\left(\hat{x}_{\lambda}\right) f\left(\hat{x}_{\lambda}\right)$. Then 
$\varphi(\gamma) f_{\lambda}=f_{\lambda}$ for all $\gamma \in U_{\lambda} . F_{\lambda}=\left\{v \in F \mid \varphi(\gamma) v=v\right.$ for all $\left.\gamma \in U_{\lambda}\right\}$. Then the mapping

$$
f \rightarrow\left(f_{1}, \cdots, f_{h}\right)
$$

gives an isomorphism of $M_{2}(\mathcal{O})$ into $F_{1} \times \cdots \times F_{h}$.

Using this isomorphism, we consider $\rho$ as giving a representation of $R\left(U, D_{N}\right)$ on $F_{1} \times \cdots \times F_{h}$. Specifically for $\xi \in R\left(U, D_{N}\right)$, we can represent $R(\xi)$ as the matrix

$$
R(\xi)=\left(\begin{array}{ccc}
\rho_{11}(\xi) & \cdots & \rho_{1 h}(\xi) \\
\vdots & \vdots & \vdots \\
\rho_{h 1}(\xi) & \cdots & \rho_{h h}(\xi)
\end{array}\right)
$$

where $\rho_{\lambda \mu}(\xi)$ is the linear map of $F_{\mu}$ to $F_{\lambda}$ which is the composition of the canonical injection of $F_{\mu}$ into $F_{1} \oplus \cdots \oplus F_{h}$, the inverse of the isomorphism in (3.1), $\rho(\xi)$, the isomorphism in (3.1) and finally the canonical projection of $F_{1} \times \cdots \times F_{h}$. Let

$$
p_{\lambda}=e_{\lambda}^{-1} \sum_{\gamma \in U_{\lambda}} \varphi(\gamma)
$$

where $e_{\lambda}=\left|U_{\lambda}\right|$.

$p_{\lambda}$ defines a projection of $F$ into $F_{\lambda}$. Let $i_{\lambda}$ denote the canonical injection of $F_{\lambda}$ into $F$. For $\xi \in R\left(U, D_{N}\right)$, put

$$
\beta_{\lambda \mu}(\xi)=i_{\lambda} \circ \rho_{\lambda \mu}(\xi) \circ p_{\mu} .
$$

Then $\beta_{\lambda \mu}(\xi)$ is an endomorphism of $F$ and

$$
B(\xi)=\left(\begin{array}{ccc}
\beta_{11}(\xi) & \cdots & \beta_{1 h}(\xi) \\
\vdots & & \vdots \\
\beta_{h 1}(\xi) & \cdots & \beta_{h h}(\xi)
\end{array}\right)
$$

gives an endomorphism of $F^{h}=F \oplus \cdots \oplus F$ which is a matrix representation of $R(\xi)$. That is, if we choose suitable bases, there exists a non-singular $h \times h$ matrix $X$ such that

$$
X B(\xi) X^{-1}=\left(\begin{array}{cc}
C(\xi) & 0 \\
0 & 0
\end{array}\right) .
$$

If $\xi=T(n) \in R\left(U, D_{N}\right)$ we next prove that $B(T(n))$ is just the Brandt matrix $B\left(n ; N^{\prime}\right)$ defined in [5].

Theorem 3.2. For $n>0$ the matrix $B(T(n))$ defined in (3.3) is identical to Brandt matrix defined in the previous section. 
Proof. Let $\xi=U y U \in R\left(U, D_{N}\right)$. Then

$$
\rho_{\lambda \mu}(\xi)=\sum_{\alpha} \varphi_{\mu}(\alpha)
$$

where the sum is over all cosets $U_{\lambda} \hat{x}_{\lambda}^{-1} \hat{x}_{\mu} \alpha\left(\alpha \in A^{\times}\right)$contained in $U_{\lambda} \hat{x}_{\lambda}^{-1} y \hat{x}_{\lambda} U_{\lambda}$ and $\varphi_{\mu}(\alpha)$ denotes the restriction of $\varphi(\alpha)$ to $F_{\mu}$. Thus

$$
\begin{aligned}
\beta_{\lambda \mu}(\xi) & =i_{\lambda} \circ \rho_{\lambda \mu}(\xi) \circ p_{\mu}(\xi) \\
& =\frac{1}{e_{\mu}} \sum_{\alpha} \sum_{\gamma} \varphi(\alpha) \varphi(\gamma),
\end{aligned}
$$

where $\sum_{\alpha}$ is as in (3.4) and $\sum_{\gamma}$ is over all $\gamma \in U_{\mu}$. Since $\varphi(\alpha) \varphi(\gamma)=$ $\varphi(\gamma \alpha)$,

$$
\beta_{\lambda \mu}(\xi)=\frac{1}{e_{\mu}} \sum_{\eta} \varphi(\eta)
$$

where the sum is over all $\eta \in A^{\times} \cap \hat{x}_{\mu}^{-1} U u U \hat{x}_{\lambda}$. Thus

$$
\beta_{\lambda \mu}(T(n))=\frac{1}{e_{\mu}} \sum_{\xi} \sum_{\eta} \varphi(\eta)
$$

where the $\operatorname{sum} \sum_{\xi}$ is over all $\xi=U y U$ such that $y_{l} \in \mathcal{O}_{l}$ for all $l<\infty$ and $N(y)=n \bmod U(\mathbb{Z})$ and for fixed $\xi$, the $\operatorname{sum} \sum_{\eta}$ is as in (3.5).

We claim

$$
\beta_{\lambda \mu}(T(n))=\frac{1}{e_{\mu}} \sum_{\eta} \varphi(\eta)
$$

where the sum is over all $\eta \in I_{\mu}^{-1} I_{\lambda}$ with $N(\eta)=n N\left(I_{\lambda}\right) / N\left(I_{\mu}\right)$. We recall that $I_{\lambda}=\mathcal{O} \hat{x}_{\lambda}$ and $N\left(I_{\lambda}\right)$ is the unique positive rational number in the coset $n\left(\hat{x}_{\lambda}\right) U(\mathbb{Z})$.

Assume that $\eta \in I_{\mu}^{-1} I_{\lambda}$ with $N(\eta)=n N\left(I_{\lambda}\right) / N\left(I_{\mu}\right)$. Then letting $\hat{x}_{\lambda}=\left(x_{\lambda_{l}}\right)$, etc, we have $\eta \in I_{\mu}^{-1} I_{\lambda}=\hat{x}_{\mu}^{-1} \mathcal{O} \hat{x}_{\lambda}$ so for all $l<\infty \eta=$ $\hat{x}_{\mu}^{-1} y_{l} \hat{x}_{\lambda}$ for some $y_{l} \in \mathcal{O}_{l}$ where $N\left(y_{l}\right)=n \bmod \mathbb{Z}_{l}^{\times}$. Thus letting $y=\left(y_{l}\right)$, we have $N(y) \in n \bmod U(\mathbb{Z})$. and $\eta \in A^{\times} \cap \hat{x}_{\mu}^{-1} U y U \hat{x}_{\lambda}$.

Conversely if

$$
\eta \in A^{\times} \cap \hat{x}_{\mu}^{-1} U y U \hat{x}_{\lambda},
$$

where $y_{l} \in \mathcal{O}_{l}$ for all $l<\infty$ and $n(y)=n \bmod U(\mathbb{Z})$, we have $\eta \in$ $\hat{x}_{\mu}^{-1} \mathcal{O} \hat{x}_{\lambda}=I_{\mu}^{-1} I_{\lambda}$ with $N(\eta) \equiv N\left(\hat{x}_{\mu}^{-1}\right) N(y) N\left(\hat{x}_{\lambda}\right) \equiv n N\left(I_{\lambda}\right) / N\left(I_{\mu}\right)$ $\bmod U(\mathbb{Z})$. Since the double coset $U \eta U$ is uniquely determined by $\eta$, we see that the two sums are identical. 
Thus $B_{\lambda \mu}(T(n))$ equals the $\lambda \mu$ th the entry of Brandt matrix which shows that the matrices $B(T(n))$ and $B(n)$ are identical.

\section{Equalities between zeta functions}

By Theorem 3.2, we are able to define a zeta function using Brandt matrices because Brandt matrices are the representatives of Hecke operators. Let $\mathcal{O}\left(L_{i}, \nu\right)=\mathcal{O}_{i}\left(q p^{\nu}\right)$ be an order of a quaternion algebra $A$ of level $N_{i}$. Then

$$
\zeta\left(A, \mathcal{O}_{i}\left(q p^{\nu}\right)\right)=\sum_{n=1}^{\infty} B\left(n ; \mathcal{O}_{i}\left(q p^{\nu}\right)\right) n^{-s} .
$$

This definition of zeta function on a quaternion algebra is similar to that of [7]. By the definition of a Brandt matrix, it is clear that $\zeta(s)$ is a matrix valued function. For two matrix valued functions $f(s)$ and $g(s)$, $f(s)$ and $g(s)$ will be identified if there exists a nonsingular matrix $M$ such that $M f(s) M^{-1}=g(s)$. As in [7], we will use the trace formula to find some equalities between zeta functions on the orders in certain quaternion algebras.

It is well known that there are two ramified nonisomorphic quadratic extension fields of $\mathbb{Q}_{p}$. For the notational convenience, let $B\left(n ; \mathcal{O}_{i}\left(q p^{\nu}\right)\right)=$ $B\left(\mathcal{O}_{i}\left(q p^{\nu}\right)\right)$.

Proposition 4.1. Let $A$ be a quaternion algebra ramified precisely at a finite prime $q$ and $\infty$ and let $\mathcal{O}\left(L_{i}, \nu\right)=\mathcal{O}_{i}\left(q p^{\nu}\right)$ be an order of a quaternion algebra $A$ of level $N_{i}$ for $i=1,2$ and 3 . We assume that $L_{1}$ is the unramified quadratic extension field of $\mathbb{Q}_{p}$ and $L_{2}, L_{3}$ are the two nonisomorphic ramified quadratic extension field of $\mathbb{Q}_{p}$. Then we have

$$
\begin{aligned}
& 2 \operatorname{tr} B\left(\mathcal{O}_{1}\left(q p^{2 n+1}\right)\right)-2 \operatorname{tr} B\left(\mathcal{O}_{1}\left(q p^{2 n-1}\right)\right) \\
= & \operatorname{tr} B\left(\mathcal{O}_{2}\left(q p^{2 n+1}\right)\right)-\operatorname{tr} B\left(\mathcal{O}_{2}\left(q p^{2 n}\right)\right)+\operatorname{tr} B\left(\mathcal{O}_{3}\left(q p^{2 n+1}\right)\right)-\operatorname{tr} B\left(\mathcal{O}_{3}\left(q p^{2 n}\right)\right) .
\end{aligned}
$$

Proof. See [4].

Let $\mathcal{O}\left(q ; L_{i}, \nu, \tilde{\psi}\right)=\mathcal{O}_{i}\left(q p^{\nu}, \tilde{\psi}\right)$ be a special order of a quaternion algebra of level $N_{i}$ with a character $\tilde{\psi}$. In [6], the Brandt matrix with this special order in a quaternion algebra is defined. For the details, see [3] or [6].

Proposition 4.2. Let $k$ be an integer $\geq 2$ satisfying $\psi(-1)=(-1)^{k}$. We assume that $L_{1}$ is the unramified quadratic extension field of $\mathbb{Q}_{p}$ and 
$L_{2}, L_{3}$ are the two nonisomorphic ramified quadratic extension field of $\mathbb{Q}_{p}$. Then

$$
\begin{aligned}
& 2 \operatorname{tr} B\left(\mathcal{O}_{1}\left(q p^{2 n+1}\right), \tilde{\psi}\right)-2 \operatorname{tr} B\left(\mathcal{O}_{1}\left(q p^{2 n-1}\right), \tilde{\psi}\right) \\
= & \operatorname{tr} B\left(\mathcal{O}_{2}\left(q p^{2 n+1}\right), \tilde{\psi}\right)-\operatorname{tr} B\left(\mathcal{O}_{2}\left(q p^{2 n}, \tilde{\psi}\right)\right)+\operatorname{tr} B\left(\mathcal{O}_{3}\left(q p^{2 n+1}\right), \tilde{\psi}\right) \\
& -\operatorname{tr} B\left(\mathcal{O}_{3}\left(q p^{2 n}\right), \tilde{\psi}\right) .
\end{aligned}
$$

Proof. See Lemma 6.15 in [3].

In the following theorem, $X \oplus 2 Y$ means the direct sum of $X$ and two copies of $Y$, which is similar to the direct sum of zeta functions in [8].

Theorem 4.3. Let $A$ be a quaternion algebra ramified precisely at a finite prime $q$ and $\infty$ and let $\mathcal{O}\left(L_{i}, \nu\right)=\mathcal{O}_{i}\left(q p^{\nu}\right)$ be an order of a quaternion algebra $A$ of level $N_{i}$ for $i=1,2$ and 3 . We assume that $L_{1}$ is the unramified quadratic extension field of $\mathbb{Q}_{p}$ and $L_{2}, L_{3}$ are the two nonisomorphic ramified quadratic extension field of $\mathbb{Q}_{p}$. Then we have

$$
\begin{aligned}
& 2 \zeta\left(A, \mathcal{O}_{1}\left(q p^{2 n}\right)\right) \oplus \zeta\left(A, \mathcal{O}_{2}\left(q p^{2 n-1}\right)\right) \oplus \zeta\left(A, \mathcal{O}_{3}\left(q p^{2 n-1}\right)\right) \\
= & \zeta\left(A, \mathcal{O}_{2}\left(q p^{2 n}\right)\right) \oplus 2 \zeta\left(A, \mathcal{O}_{1}\left(q p^{2 n-2}\right)\right) \oplus \zeta\left(A, \mathcal{O}_{3}\left(q p^{2 n}\right)\right) .
\end{aligned}
$$

Proof. This is immediate from Proposition 4.1.

Theorem 4.4. Assume that $A$ is a quaternion algebra of discriminant $d$. Let $\mathcal{O}\left(L_{i}, \nu\right)=\mathcal{O}_{i}\left(q p^{\nu}\right)$ be an order of a quaternion algebra $A$ of level $N_{i}$ for $i=1,2$ and 3 . We assume that $L_{1}$ is the unramified quadratic extension field of $\mathbb{Q}_{p}$ and $L_{2}, L_{3}$ are the two nonisomorphic ramified quadratic extension field of $\mathbb{Q}_{p}$. Then

$$
\begin{aligned}
& 2 \zeta\left(A, \mathcal{O}_{1}\left(q p^{2 n+1}\right), \tilde{\psi}\right) \oplus \zeta\left(A, \mathcal{O}_{2}\left(q p^{2 n}\right), \tilde{\psi}\right) \oplus \zeta\left(A, \mathcal{O}_{3}\left(q p^{2 n}\right), \tilde{\psi}\right) \\
= & \zeta\left(A, \mathcal{O}_{2}\left(q p^{2 n+1}\right), \tilde{\psi}\right) \oplus 2 \zeta\left(A, \mathcal{O}_{1}\left(q p^{2 n-1}\right), \tilde{\psi}\right) \oplus \zeta\left(A, \mathcal{O}_{3}\left(q p^{2 n+1}\right), \tilde{\psi}\right) .
\end{aligned}
$$

Proof. This is immediate from Proposition 4.2.

\section{References}

[1] M. Eichler, The basis problem for modular forms and the traces of Hecke operators. Springer-Verlag, Lecture Notes in Math., Vol.320, pp. 75-151, 1972.

[2] R. Godement, Les fonctions $\zeta$ des algebras simples I, II, Seminaire Bourbaki, $1958 / 1959$.

[3] H. Hijikata, A. Pizer, T. Shemanske, The basis problem for modular forms on $\Gamma_{0}(N)$ Memoirs AMS, Vol.32 No.418, November, 1989.

[4] S. Jun, The equalities between Brandt matrices on primitive orders, preprint.

[5] S. Jun, On the representatives of modular forms on $\Gamma_{0}(N)$, J. Algebra, Vol.64, pp. 340-390, 1980. 
[6] A. Pizer, An algorithm for computing modular forms by theta series, J. KMS, Vol.34 No.4, pp. 808-816, 1997.

[7] H. Shimizu, On the traces of Hecke operators, J. Fac. Sci. Univ. Tokyo, Vol.10, pp. 1-19, 1963.

[8] H. Shimizu, On zeta functions of quaternion algebra, Ann. of Math., Vol.81, pp. 166-193, 1965.

[9] G. Shimura, "Introduction to the arithmetic theory of automorphic functions", Princeton Univ Press, Princeton, N.J., 1971.

[10] T. Tamagawa, On $\zeta$ functions of a division algebra, Ann. of Math. Vol.77, pp. 387-405, 1963.

Insuk kim

Department of Mathematics Education, Wonkwang University, Iksan, Jeonbuk 570-749, Korea.

E-mail: iki@wonkwang.ac.kr

Sungtae Jun

Division of Mathematics and Computer Science, Konkuk University, Choongju, Choongbuk 380-151, Korea.

E-mail: sjun@kku.ac.kr 